\title{
Application of classification neural networks for identification of damage stages of degraded low alloy steel based on acoustic emission data analysis
}

\author{
Joanna Krajewska-Śpiewak ${ }^{1}$ - Igor Lasota ${ }^{2} \cdot$ Barbara Kozub $^{3}$
}

Received: 13 February 2020 / Revised: 27 August 2020 / Accepted: 31 August 2020

(c) The Author(s) 2020

\begin{abstract}
The paper presents the influence of low alloy steel degradation on the acoustic emission (AE) generated during static tension of notched specimen. The material was cut from a technological pipeline long-term operated in the oil refinery industry. Comparative analysis of AE activity generated by damage process of degraded and new material has been carried out. The different AE parameters were used to detect different stages of fracture process of low alloy steel under quasi-static tensile test. Neural networks with three layers were created with Broyden-Fletcher-Goldfarb-Shanno learning algorithm for a database analysis. The different AE parameters were included in the input layer. Classification neural networks were created in order to determine the stages of material degradation. The results obtained from the carried out studies will be used as the basis for new methodology development of the assessment of the structural condition of in-service equipment.
\end{abstract}

\section{Introduction}

Low alloy steels with different chemical composition are widely used in pressure vessels structures, operated in the oil refining and petrochemical processing. To work for decades, the pressure equipment needs to withstand extreme conditions such as high/low temperatures, time-varying pressure load and influence of aggressive media. Long-term application of equipment under extreme conditions can cause defects initiation and growth in the microstructure of the materials which can damage the equipment or cause the failure of the entire industrial unit.

Joanna Krajewska-Śpiewak

joanna.krajewska-spiewak@pk.edu.pl

Igor Lasota

lyasota.igor@gmail.com

Barbara Kozub

barbara.kozub@mech.pk.edu.pl

1 Faculty of Mechanical Engineering, Production Engineering Institute, Cracow University of Technology, Al. Jana Pawła II 37, 31-864 Kraków, Poland

2 PKN Orlen S.A., Chemików 7, 09-411 Płock, Poland

3 Faculty of Materials Science and Physics, Institute of Material Engineering, Cracow University of Technology, Al. Jana Pawła II 37, 31-864 Kraków, Poland
Therefore, the development of new, non-destructive methodologies which allow to assess the condition of materials after long-term use in the refinery industry is very important. Development of such methodologies is possible only by complex laboratory investigations.

This research is aimed at using acoustic emission data analysis for the identification of plastic deformation and fracture processes in low alloy steel under quasi-static loading. The tested material came from the long-term operated seamless furnace coil pipe of the crude oil distillation unit. Tensile tests of notched flat specimens were performed with simultaneous registration of AE signal. Microstructural damage processes were correlated by in situ examinations with the use of portable optical light microscopy. The results of the studies will be "basis" of the methodology for material condition evaluation of in-service pressure vessels. The conducted investigations were financed by the National Centre for Research and Development by the LIDER VII Project.

$\mathrm{H}$. Dunegan with colleges developed one of the first correlation between AE activity and the fracture mechanisms of different materials $[1,2]$. Since then, the AE behaviour during various stages of fracture processes is systematically studied with advances of progress in measuring systems and signal analysis algorithms [3]. The different investigations of acoustic emission behaviour during deformation and fracture processes of Armco iron [4, 5], carbon steels $[6,7]$ and stainless steels $[8,9]$ were carried out. Acoustic 
emission signals with the typical parameters are recorded at different stages of the tensile loading process $[10,11]$. The dislocation movement at ferrite grain boundaries is an $\mathrm{AE}$ source during the early stage of plastic deformation. Then, a significant increase of AE activity is observed as a result of the intense slip band motion, when material achieves yield point [12]. The low-energy signals can be generated by the increase of dislocation number and their unpinning from the Cottrell atmosphere. The process of parallel movement of high-density dislocations (Lüders bands) may emit the latter high-energy signals $[13,14]$. To sum it up, the rapid increase of AE activity takes place when the material reaches the yield point but this activity decreases exponentially with the next loading $[12,15]$.

Slightly different acoustic emission behaviour is observed during deformation and fracture of stainless steel compared to carbon steel. This is linked to the chromium carbides which are present in the stainless steel microstructure. The influence of the carbides content in aged austenitic stainless steels on AE activity is described in detail by authors [16, 17]. Generally, the number of AE events during fracture of the thermally aged material is significantly lower than in annealed ones. This effect is a result of blocking of dislocation movement by diffusion of chromium or other chemical elements $[18,19]$. Besides, additional AE activity is generated by increased grain boundary brittle cracking during the deformation of the aged stainless steel. In accordance with the literature, the AE method is highly sensitive to the identification of each stage of the material fracture process.

Neural networks are commonly applied in material science, mainly for prediction of: phase transformation behaviours, material properties or for optimization of processing parameters. Neural networks use a hypothetical learning process in the cognitive system and brain neurological functions. The learning process is based on existing data. Regression neural networks can predict the values of new observations based on other observations made on the same or different variables during the learning process. In case of the classification neural networks, a set of elements is divided into groups which include elements that differ but have properties that distinguish a given group (to some extent "similar elements"). Elements of individual classes may differ, except for those properties on the basis of which their classification is based. Depending on the available data, the classification can be divided into reference and no- reference classification. The reference classification uses the characteristics of the classes from which the analysed data come from. This approach is also called supervised learning (learning with a teacher). The main goal of the learning process is to achieve adequate quality classification of data that was not used during model learning also known as test data. Fitting the model to the training data is also important in classification neural networks. It should be emphasized that there are only a few scientific publications about the $\mathrm{AE}$ data analysis of low alloy steel fracture processes with the use of artificial neural networks.

\section{Materials and methods}

Two materials were used in carried out studies. The first group of simples was obtained from 6 years old seamless coil pipe of crude oil distillation unit ("used material"). It was made out of A335 grade P5 ferritic alloy steel. The second group of simples was obtained from brand-new A335 grade P5 tube with diameter of $163 \mathrm{~mm}$ ("unused material") [20]. The chemical composition all of materials is comparable and can be seen in Table 1.

Light microscopy was used to conduct metallographic examinations. It can be observed (Fig. 1) that the microstructure of the used material is significantly different from the brand-new material. The microstructure is considerably heterogeneous with directed grain growth and consists of areas with a patchy distribution of carbides. There are also zones depleted in carbides (Fig. 1b). Long term overheating, in the range $>600^{\circ} \mathrm{C}$, was the cause of degradation changes to the morphology of the used pipe's microstructure.

AE signals were recorded during the static tensile tests conducted on notched flat specimens (Fig. 2a). Electroerosion method (tip radius- $-0.05 \mathrm{~mm}$ ) was used to create the notch at the one side of the specimen. The metallographic sections were prepared on the flat side of specimens (between shoulders) for in-situ monitoring of the microstructure during the tensile test.

The specimens were axially loaded on a testing machine with hydraulic serrated grips. Acoustic emission was recorded by AMSY-6 multi-channel AE measurement system (with ASIP-2/A channel type). Testing station (Fig. 2b) consists of: VS150-M and VS75-V type resonance sensors (resonance frequency of $150 \mathrm{kHz}$ and $75 \mathrm{kHz}$ ) and $34 \mathrm{~dB}$ external preamplifiers. The AE channels were calibrated by 2H/0.5 mm Hsu-Nielsen source according to the PN-EN 13544:2011 standard [21].

\section{Results and discussion}

\subsection{Identification of damage process stages by AE method}

The representative results of notched specimen tensile tests such as stress-displacement curves with AE signal parameter distribution for both samples are shown in Figs. 3. The AE signals with high values of burst peak amplitudes were observed almost from the beginning of the tensile test of notched specimens while a rapid increase of AE activity was 
recorded only near the yield point in the case of the A335 grade $\mathrm{P} 5$ unnotched flat samples, presented in article [6].

The detailed analysis of AE signal parameters (max. amplitude distributions, duration/maximum amplitude (DA) and rise time/maximum amplitude (RA) parameters [22], as well as Historic Index (HI) MONPAC criterion [23]) allowed to identify each mechanism of deformation and fracture processes during the tensile test of A335 P5 steel notched specimens (Fig. 3).

The AE signals with high values of burst peak amplitudes were recorded almost from the beginning of the tensile test of notched specimens, cut from new and used material. The first signals were as result of the processes of active dislocation formation in ferrite grains located near the notch tip. It is observed for both materials after exceeding a stress value approx. 80-100 MPa (black point number "1" in Fig. 3h). This fact has been also validated by in situ observations of material microstructure changes near the notch tip with the use of portable light microscope. In addition, the finite element method (FEM) calculations (with nonlinear analyses) showed a stress concentration in the specimen notch tip (Fig. 4a). The values of these stresses concentration exceed material conventional yield strength (for A335 grade P5 unused steel- $\mathrm{Re}=284 \mathrm{MPa}$ ) for the abovementioned load conditions.

The 3D finite element model of the specimen with side notch was built. The mesh with tetragonal finite elements was used in the model. The mesh was concentrated around the notch tip area to improve the accuracy of the FEM analysis. The nonlinear plastic analysis was carried out. The real stress-strain curves, and mechanical as well as physical properties of the material were implemented into the model. The materials' mechanical properties were obtained during laboratory tensile tests (Table 1). The model was fixed and loaded by axial force identically as during the real tensile test of the specimen.

The next stage of the fracture process was crack initiation at the notch tip, which was also confirmed by the FEM calculations and SEM fractography investigations (Fig. 4b, c). The crack initiation caused an active increase of the $\mathrm{AE}$ parameter values. In the cases of both material, the crack was initiated after exciting approx. 200-220 MPa (black points number "2" in Fig. 3g, h). This load exceeds material ultimate strength (for A335 grade P5 steel- $\mathrm{Rm}=515 \mathrm{MPa}$ ) at the notch tip according to the FEM analysis (Fig. 4b).

The materials plastic deformation in the work cross-section of specimens (offset yield point in the stress-displacement curves) was observed in the next stage of the material damage process. The highest $\mathrm{AE}$ activity is generated at this moment during tensile of unused material. This activity for signals recorded by VS75-V sensors was significantly higher than those recorded by type VS150-M sensors. This fact confirmed that acoustic emission sensors with a resonance 
Fig. 1 The microstructure of unused (a) and used (b) A335 grade P5 steel (magnification $600 \times)$ a

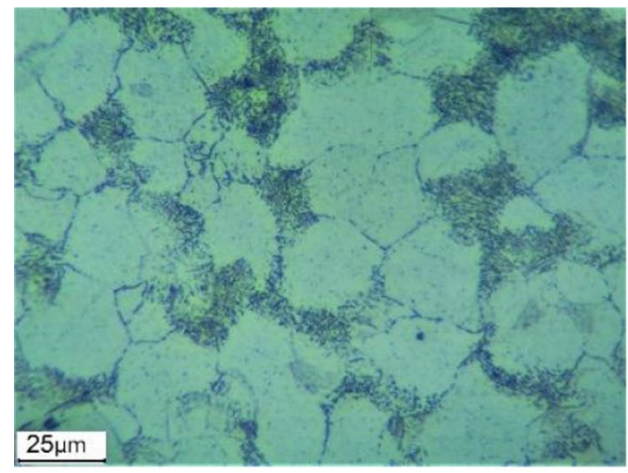

b



Fig. 2 Technical drawing of tested specimen (a) and diagram of the testing station (b) a

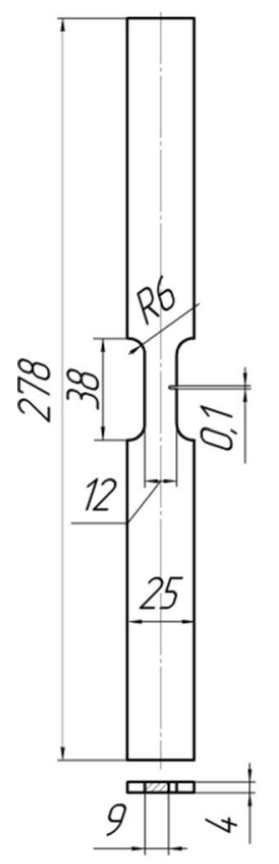

b

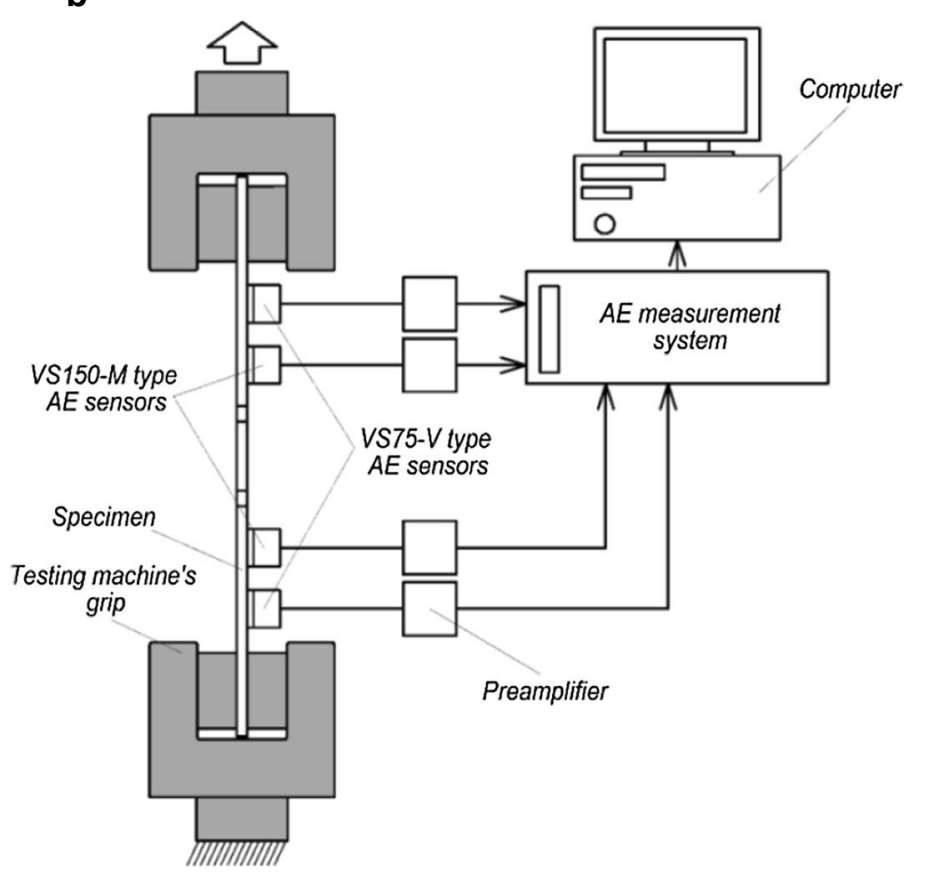

frequency of $75 \mathrm{kHz}$ are more sensitive to the plastic deformation of A335 P5 grade steel.

The last stage was a rapid start of macro-cracks with critical growth. The increase of AE activity was also noted during transition of crack propagation process to the critical stage. In degraded material, these processes generated $\mathrm{AE}$ signals with maximum amplitudes up to $95 \mathrm{~dB}$.

\subsection{Application of classification neural networks}

Based on the previous studies, neural networks were used to classify stages of material degradation based on registered AE parameters such as burst peak amplitude, DA, RA, HI and energy. Figure 5 represents allocation of the AE signal DA parameter for individual stages of material degradation during tensile test. These grouped individual stages were used in neural networks. A preliminary analysis of the AE signal parameters was made. In all cases, the greatest differences for the analysed stages of the material degradation were obtained for DA and Energy parameters. Therefore, additional parameter in form of the ratio of DA/E parameters was introduced to the input layer of neural networks.

A standard three layers (input, hidden and output) network structure was created. The input layer consists of all considered AE signal parameters including DA/E. In order to find the optimal classification networks, simulations have been carried out based on the following variable properties:

Fig. 3 Localization of AE events $(\mathbf{a}, \mathbf{b})$ as well as stress-displacement curves with AE signal amplitude (c, d), DA parameter $(\mathbf{e}, \mathbf{f})$ and Historic Index signal parameter $(\mathbf{g}, \mathbf{h})$ distributions for A335 grade P5 used $(\mathbf{a}, \mathbf{c}, \mathbf{e}, \mathbf{g})$ unused $(\mathbf{b}, \mathbf{d}, \mathbf{f}, \mathbf{h})$ recorded by type VS150-M resonant sensors 
a

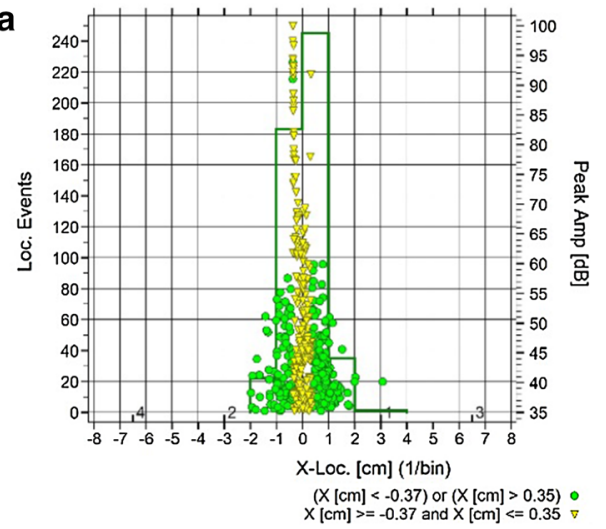

C

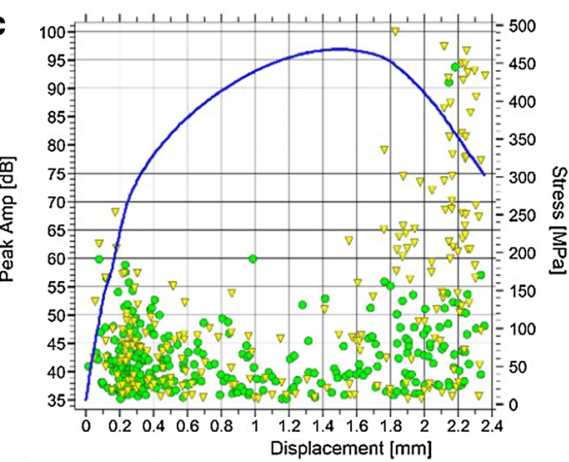

- $(X[\mathrm{~cm}]<-0.37)$ or $(X[\mathrm{~cm}]>0.31$,

e
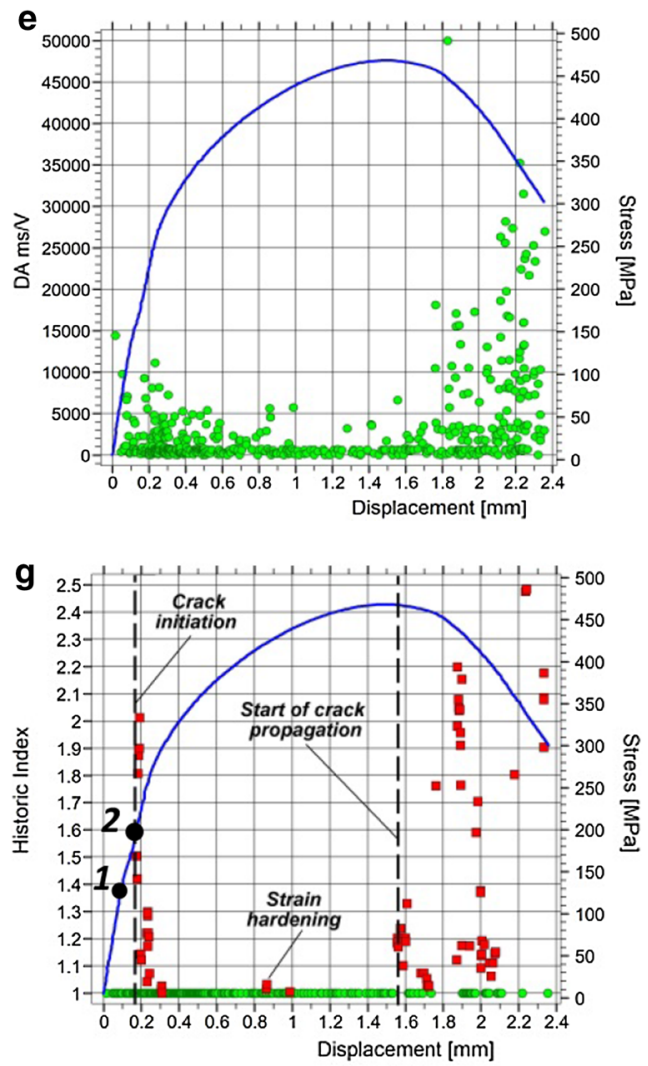


$-(X[\mathrm{~cm}]<-0.35)$ or $(X[\mathrm{~cm}]>0.35)$
$\forall X[\mathrm{~cm}]>=-0.35$ and $X[\mathrm{~cm}]<=0.35$

a



b

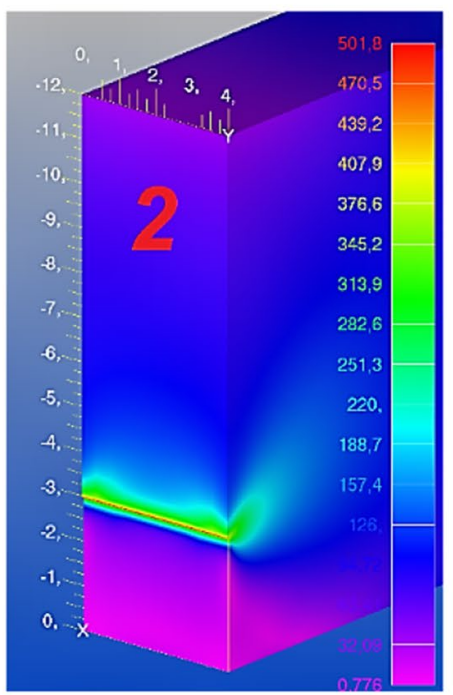

C


d

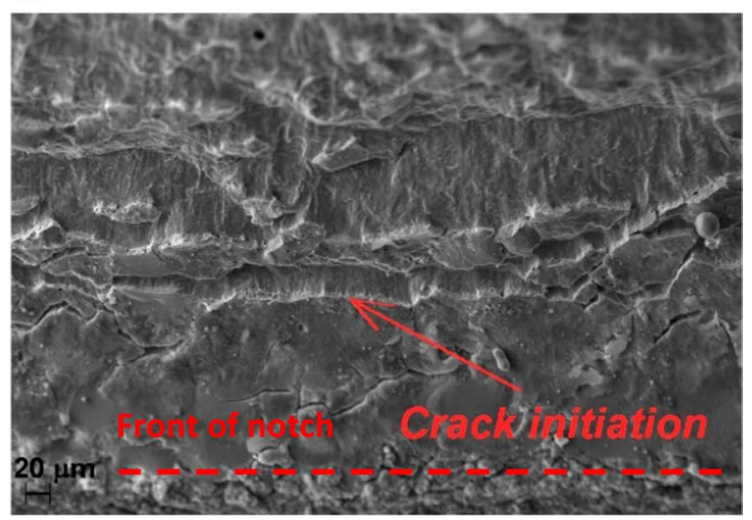

Fig. 4 Von Mises stress distribution [MPa] (a, b) in specimen for different loading levels (according to points 1, 2 from Fig. 3), and fractography images of fracture cross-section of the specimen $(\mathbf{c}, \mathbf{d})$

Fig. 5 The functioning of applied classification neural networks

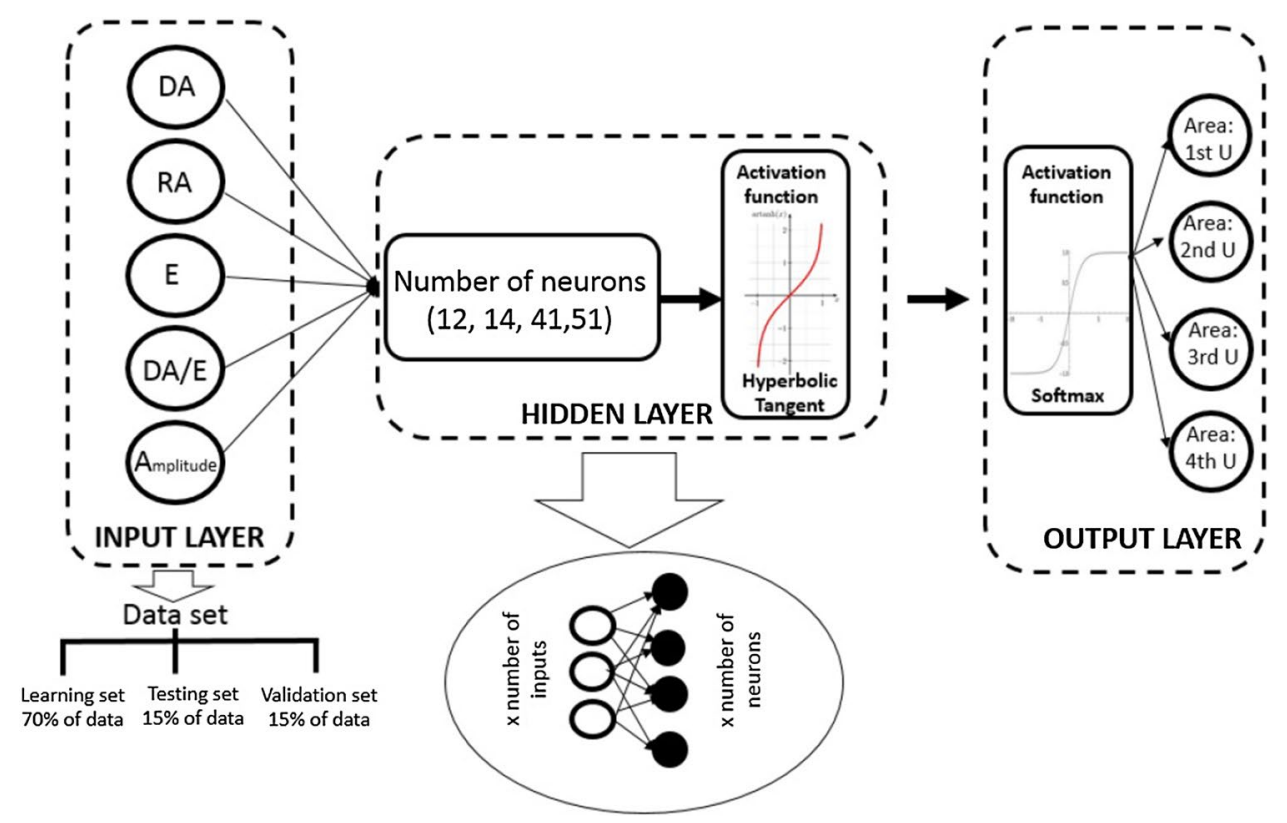

type of activation function (linear, sine, tangent, logistic, exponential).

The smallest matching error was obtained for a one-way multi-layer neural network (MLP) with one hidden layer 
Table 2 Neural networks architectures of used and unused samples

\begin{tabular}{|c|c|c|c|c|c|c|c|c|}
\hline $\begin{array}{l}\text { Material/sen- } \\
\text { sor's resonance } \\
\text { frequency }\end{array}$ & Network & $\begin{array}{l}\text { Quality } \\
\text { of learn- } \\
\text { ing }\end{array}$ & Quality of testing & $\begin{array}{l}\text { Quality of } \\
\text { validation }\end{array}$ & $\begin{array}{l}\text { Learning algo- } \\
\text { rithm }\end{array}$ & Error function & $\begin{array}{l}\text { Activa- } \\
\text { tion (hid- } \\
\text { den) }\end{array}$ & Activation (output) \\
\hline Used/75 kHz & MLP 5-41-4 & 84.81 & 62.50 & 37.50 & BFGS 75 & Entropy & Tanh & Softmax \\
\hline Used/150 kHz & MLP 5-12-4 & 80.60 & 84.62 & 30.77 & BFGS 82 & Entropy & Tanh & Softmax \\
\hline Unused/75 kHz & MLP 5-51-4 & 98.00 & 80.00 & 60.00 & BFGS 73 & Entropy & Tanh & Softmax \\
\hline Unused $/ 150 \mathrm{kHz}$ & MLP 5-15-4 & 89.36 & 70.00 & 20.00 & BFGS 44 & Entropy & Tanh & Softmax \\
\hline
\end{tabular}
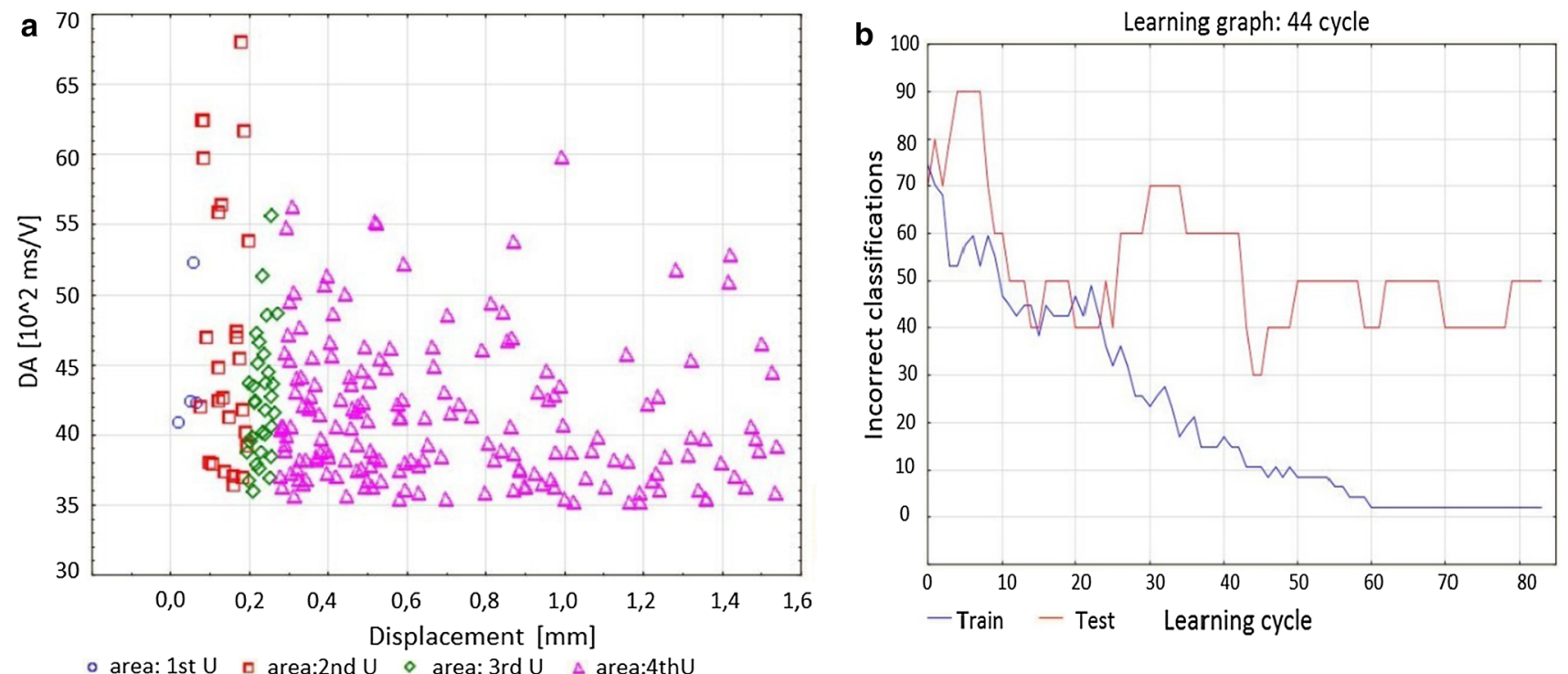

Fig. 6 a Allocation of DA parameter (VS 150-M sensor type). b Learning graph of unused samples (VS150-M sensor type)

with different number of hidden neurons. Individual neural networks were generated for all samples. The diagram in Fig. 5 shows how the applied neural networks operate.

Each input variable is multiplied by its corresponding weight (the weight values are changed during the learning process and can be delayed or stimulating) at the time the neural network is started. Then, the sum of the input features is passed to the activation function, which is a mathematical relationship determining the signal transmission from earlier neurons.

The selection of the activation function depends on the type of problem (regression or classification problem). Nonlinear functions are most commonly used because neurons with such characteristics have the greatest ability to learn and allow smooth mapping of the relationship between the input and output characteristics. Therefore, a continuous value instead of a logical value is obtained. Output neurons are equipped with an activation function that varies depending on the situation, it can be an identity function (in regression problem in form of tanh in hidden layer) or a different one for classification tasks (eg. softmax). The softmax function is adapted to the classification problems in which the representation of the "one-with-N" type output variable is used. The sum of activation of individual neurons of the output layer with the Softmax activation function is equal to 1 , hence the activation of neurons is interpreted as the probability of occurrence of a given class. After all calculations have been carried out, the output of the entire network appears. The architecture of networks is presented in Table 2.

One of the quasi-Newtonian methods in form of the Broyden-Fletcher-Goldfarb-Shanno (BFGS) algorithm was used in learning process of the networks. A symmetrical function of S-shaped hyperbolic tangent ( $\tanh )$ was used for signal transmission. The outputs of neurons with this activation function are centered around zero. Entropy was used as an error function, which means that the data comes from the exponential distribution family and provide direct probabilistic interpretation of the network outputs. The choice of error function is directly related to the determination of the softmax function as an activating function of the output layer. Data from the hidden layer were additionally 


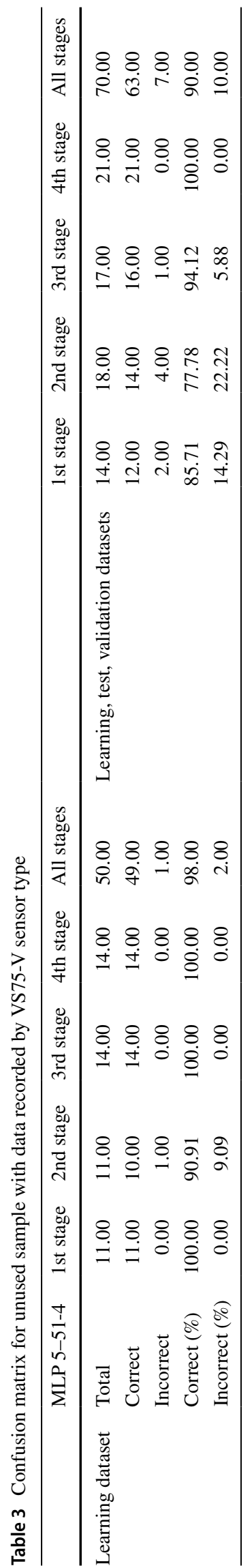

normalized in such a way that the sum of the activation of the whole layer was equal to 1 . In the case of classification tasks the softmax function is the most common activating function of outputs.

Obtained learning cycles show similar tendency. The learning graph of unused sample is shown in Fig. 6b. It can be observed that classification errors made by networks in the initial stage of learning are rapidly decreasing. Then, as the network is learning and its information is absorbed, the network at the right time (specific epoch), achieves the smallest value of the error and the learning process ends. The network can be verified. Data introduced to the network was divided into three sets: validation, test and learning. These sets were chosen arbitrarily, always in the same proportions: $70 \%$ learning and $15 \%$ validation and test set. The validation set is used to check whether the network is not learned by heart, and it is possible to stop the learning process, if necessary. The data in the test set is responsible for the final test of the quality of the network.

Based on the data from training set it can be seen that the network is very good at proper classification. It is confirmed by the convex shape of obtained curves. It is also confirmed by the generated confusion matrix for the training set (Table 3) which provides information about the percentage of correctly classified data.

To check how individual networks cope with data that is not involved in the learning process, new cases have been added to the network (so-called 'missing' data) based on previously conducted network learning process based on a learning, validation and test set. The number of entered missing data was different for all networks, which was caused by the varied number of registered AE signal parameters.

The learning process ran smoothly in all generated networks. In case of networks for unused samples, entered data was correctly allocated in all cases. Networks generated for used samples had problems with the correct classification. This may be due to the relatively high data disproportion in individual stages (e.g. 2nd stage - 20 cases, 3rd stage44 cases in used 75). Global sensitivity analysis was also determined to check the significance of the input parameters (Table 4).

The global analysis shows that all input parameters provide relevant information to neural networks for proper learning, validation and testing, as evidenced by the received values which are greater than 1 . It can be seen that the role of the additional parameter is not indifferent during learning. In three cases the role of parameter DA/E is greater than HI parameter.

It should be noted that classification neural networks, with the right architecture, show good learning ability based on AE signal parameters and can be used to determine the degree of material degradation. 
Table 4 Sensitivity analysis of samples

\begin{tabular}{lllllll}
\hline Material & $\begin{array}{l}\text { Sensor's resonance } \\
\text { frequency }\end{array}$ & AE parameters & & & \\
\hline Used & $75 \mathrm{kHz}$ & Peak Amp & DA & Energy & DA/E & HI \\
& & 3.9834 & 3.5762 & 1.5028 & 1.2657 & 1,1993 \\
& \multirow{2}{*}{$150 \mathrm{kHz}$} & Peak amp & DA & HI & DA/E & Energy \\
& & 6.9703 & 5.3747 & 4.1911 & 2.9455 & 2.3742 \\
Unused & $75 \mathrm{kHz}$ & $\mathrm{DA}$ & Peak amp & DA/E & Energy & HI \\
& & 18.9130 & 16.8936 & 9.8219 & 3.6663 & 3.6121 \\
& \multirow{2}{*}{$150 \mathrm{kHz}$} & $\mathrm{DA}$ & Peak Amp & Energy & DA/E & Hi \\
& & 6.4036 & 4.0689 & 3.2662 & 2.6981 & 2.5474 \\
\hline
\end{tabular}

\section{Conclusions}

Detection of each stage of damage process was possible with AE method during static tension testing of notched specimens made out of ferritic alloy A335 P5. The AE generated by the damage processes was well correlated with in-situ observation of microstructural changes as well as fractography examinations and FEM simulations of stress/strain fields. The detailed analysis of AE signals parameters generated during the tensile testing of notched specimens allowed identification of each stage/mechanism of the deformation and fracture processes: material plastic deformation and crack initiation near the notch tip; deformation and strain hardening in the whole work cross section of specimens; stable crack growth. Carried out research confirmed that $\mathrm{AE}$ sensors with a resonance frequency of $75 \mathrm{kHz}$ are more sensitive to plastic deformation of steel, while VS150-M type sensors with a frequency of $150 \mathrm{kHz}$ have a higher sensitivity to crack initiation and propagation processes. Classification neural networks, with appropriate architecture, show good learning ability based on AE signal parameters and can be used to determine stages of material degradation. However, this does not change the fact that from the point of view of reservoir retention, the interesting zones represent a small fraction of all recorded AE data. Therefore, in order to achieve higher learning and validation quality of neural networks, it is recommended to carry out additional tests to provide the network with more learning and validation cases that should improve the classification process of neural networks.

Acknowledgements Authors would like to thank dr hab. J. Schmidt and msc. eng. T. Ryncarz for help in the implementation of researches. This study was supported by the project of the LIDER VII Programme entitled "The new comprehensive method to assess the technical condition of the pressure equipment of installation operated in the chemical and petrochemical industry, using analysis of acoustic emission signals, structure stress field and the degradation state of the material" financed by the National Centre for Research and Development of Poland.
Open Access This article is licensed under a Creative Commons Attribution 4.0 International License, which permits use, sharing, adaptation, distribution and reproduction in any medium or format, as long as you give appropriate credit to the original author(s) and the source, provide a link to the Creative Commons licence, and indicate if changes were made. The images or other third party material in this article are included in the article's Creative Commons licence, unless indicated otherwise in a credit line to the material. If material is not included in the article's Creative Commons licence and your intended use is not permitted by statutory regulation or exceeds the permitted use, you will need to obtain permission directly from the copyright holder. To view a copy of this licence, visit http://creativecommons.org/licenses/by/4.0/.

\section{References}

1. Grosse CU, Ohtsu M. Acoustic emission testing, basics for research-applications in civil engineering. Berlin: Springer; 2008. p. 414.

2. Dunegan HL, Harris DO, Tatro CA. Fracture analysis by use of acoustic emission. Eng Fract Mech. 1968;1:105-22.

3. Ono K. Acoustic emission in materials research-a review. J AE. 2011;29:284-308.

4. Lazarev A, Vinogradov A. About plastic instabilities in iron and power spectrum of acoustic emission. J Acoust Emiss. 2009;27:144-56.

5. Almeida DM, Maia NS, Bracarense AQ, Medeiros EB, Maciel TM, Santos MA. Characterization of steel pipeline damage using acoustic emission technique. Soldagem Insp. 2007;12:55-62.

6. Lyasota I, Kozub B, Gawlik J. Identification of the tensile damage of degraded carbon steel and ferritic alloy-steel by acoustic emission with in situ microscopic investigations. Arch Civil Mech Eng. 2019;19:274-85.

7. Yang D, Yang L, Dong-ming F. Monitoring damage evolution of steel strand using acoustic emission technique and rate process theory. J Cent South Univ. 2014;21:3692-7.

8. Sahoo S, Jha BB, Sahoo TK. Acoustic emission study of deformation behaviour of sensitised 304 stainless steel. Energy Mater. 2014;9:1336-422.

9. Mukhopadhyay CK, Phaniraj C, Jayakumar T, Samuel KG. Study of tensile behaviour of thermally aged alloy D9 wrapper tubes of fast breeder reactor using acoustic emission. Strength Fract Complex. 2014;8:219-29.

10. Marsudi M. Study of acoustic emission during tensile test of mild steel plate, 8th international conference on heat transfer, fluid mechanics and thermodynamics. 2011; 11-13 
11. Zou S, Yan F, Yang G, Sun W. The identification of the deformation stage of a metal specimen based on acoustic emission data analysis. Sensors. 2017;17(4):789.

12. Akbari M, Ahmadi M. The application of acoustic emission technique to plastic deformation of low carbon steel. Phys Procedia. 2010;3:795-801.

13. Wang HW, Yu HM, Xiao HQ, Han ZY, Luo HY. Steel damage based on acoustic emission. Mater Res Innov. 2015;19:288-91.

14. Lee CS, Huh JH, Li DM, Shin DH. Acoustic emission behaviour during tensile tests of low carbon steel welds. ISIJ Int. 1999;39:365-70.

15. Penglin Z, Yuan S, Zhiqiang Z, Yaxing X. Application of acoustic emission technique in q345e steel tensile damage detection. $\mathrm{J}$ Gansu Sci. 2015;2:83-7.

16. Mukhopadhyay CK, Jayakumar T, Raj B, Ray KK. Acoustic emission during tensile deformation of pre-strained nuclear grade AISI type 304 stainless steel in the unnotched and notched conditions. J Mater Sci. 2007;42:5647-56.

17. Máthis K, Prchal D, Novotny R, Hähner P. Acoustic emission monitoring of slow strain rate tensile tests of $304 \mathrm{~L}$ stainless steel in supercritical water environment. Corros Sci. 2011;53:59-63.
18. Lee JK, Lee JH, Lee SP, Son IS, Bae DS. Acoustic emission and ultrasonic wave characteristics in TIG-welded 316 stainless steel. Met Mater Int. 2014;20:483-8.

19. Penkin AG, Terent'ev VF, Roshchupkin VV, Slizov AK, Sirotinkin VP. Acoustic emission analysis of the stages of deformation of TRIP steel. Russ Metall. 2017;4:306-11.

20. ASTM A335 Standard Specification for Seamless Ferritic AlloySteel Pipe for High-Temperature Service.

21. EN 13554 : 2011 Non-destrucitve testing - Acoustic Emission testing - General principles.

22. Ohno K, Ohtsu M. Crack classification in concrete based on acoustic emission. Constr Build Mater. 2010;24:2339-466.

23. Fowler TJ, Blessing JA, Conlisk PJ, Swanson TL. The MONPAC System, world meeting on acoustic emission. Charloue. N. Carolina, March 1989

Publisher's Note Springer Nature remains neutral with regard to jurisdictional claims in published maps and institutional affiliations. 